\title{
3 Politische Perspektiven für die Zukunft der digitalen Gesundheit
}

\author{
Maik Beermann
}

\section{Einleitung}

Die Digitalisierung ist inzwischen in der Bundesrepublik Deutschland angekommen. In allen Bereichen des Zusammenlebens spielen digitale Konzepte und Innovationen heute eine entscheidende Rolle. Von der Flexibilisierung der Arbeit in Form von Home-Office Konzepten und zeitlich- und standortunabhängigem Arbeiten, über digitalen Zugang zu Verwaltungsleistungen im Rahmen des eGovernment, bis hin zu intelligenter Datenanalyse und sensorgestützter Tierhaltung in der Landwirtschaft, die digitale Revolution ist in vollem Gange.

Der 18. Deutsche Bundestag hat auf diese besondere Entwicklung reagiert und mit dem Ausschuss für Digitale Agenda erstmals das Thema Digitalisierung in die parlamentarische Arbeit aktiv eingebunden. In enger Abstimmung mit dem Ausschuss für Verkehr und digitale Infrastruktur werden hier die Konzepte erarbeitet um den Übergang zu Deutschland 4.o auch von politischer Seite fachmännisch zu begleiten, die richtigen Rahmenbedingungen zu schaffen und rechtliche Grundlagen zu erarbeiten, um unsere Bundesrepublik zukunftsfähig aufzustellen.

Dabei sehen sich die Abgeordneten des Deutschen Bundestages tagtäglich neuen Herausforderungen gegenüber. Unsere Bundeskanzlerin hat die aktuelle Entwicklung treffend beschrieben, als sie verlauten ließ:

„Daten sind die Rohstoffe des 21. Jahrhunderts.“ 
In Zeiten von kostenfreiem Zugang zu Applikationen und Leistungen des Internets haben sich persönliche Daten zu der neuen Währung des Internets entwickelt. Dieser Verlauf hat das Wachstum von Unternehmen, wie Facebook und Alphabet, entscheidend beeinflusst. Auf persönliche Interessen zugeschnittene Werbung hat sich zu einem wichtigen Marktsegment entwickelt und verspricht Gewinne in Milliardenhöhe.

Die Vielfalt an Daten, welche heutzutage von jedem Nutzer produziert wird, bietet nicht nur Unternehmen, sondern auch dem Nutzer entscheidende Vorteile. Dennoch muss auch immer beachtet werden, was mit den eigenen Daten geschieht, wer diese hat und wie diese weiterverwendet werden. Ein umfangreicher Datenschutz und klar definierte Besitzeigenschaften für die Daten, sind somit zentrale Aufgabe der Politik.

Besonders im Bereich hoch sensitiver Daten, wie dies bei Gesundheitsdaten der Fall ist, ist es von hoher Bedeutung, den Datenschutz fest zu verankern, um Bürgerinnen und Bürger vor Datenmissbrauch zu schützen.

Welche Vor- und Nachteile die Digitalisierung im Gesundheitswesen für den einzelnen Bürger dabei hat, wird in diesem Band in vielfältiger Art und Weise geschildert. Welche gesetzlichen Rahmenbedingungen bereits bestehen, und wie die Politik auch künftig die Entwicklung neuer Konzepte begleiten sollte, soll in diesem Beitrag erläutert werden.

\section{Gesetzliche Rahmenbedingungen}

\section{Das eHealth-Gesetz}

Mit dem Gesetz für sichere digitale Kommunikation und Anwendungen im Gesundheitswesen hat der Deutsche Bundestag im Dezember 2015 die Grundlage für die Einführung digitaler Anwendungen in der bundesdeutschen Gesundheitsversorgung gelegt.

Durch das eHealth-Gesetz ist es gelungen, die Anlegung der elektronischen Patientenakte, des Medikationsplans und der Notfalldaten gesetzlich zu verankern und im Rahmen des gematik-rollouts ein sanktionsfähiges Zeitfenster zum Aufbau einer Telematik-Infrastruktur zu schaffen.

Die Einführung der elektronischen Gesundheitskarte bis Mitte 2018 ist dabei zentraler Bestandteil des Gesetzes. Durch die Implementierung von den wichtigsten Gesundheitsdaten auf der Gesundheitskarte ist der Patient damit in der Lage, behandelnde Ärzte über den eigenen medizinischen Zustand zu informieren und kann somit Wartezeiten, welche beispielsweise durch den Versand des Arztbriefes entstehen, zu umgehen.

Im Rahmen des Datenschutzes legt das eHealth-Gesetz klare Rahmenbedingungen fest, um zu gewährleisten, dass der Patient als Inhaber seiner Daten gestärkt wird. So verfügt der Patient über die Kompetenz, zu entscheiden, welche Daten aus seiner Gesundheitskarte er welchen Ärzten zur Verfügung stellen möchte. Durch die Einführung des Patientenfaches können weiterhin auch solche Daten auf der Gesundheitskarte gespeichert werden, welche der Patient selber, beispielsweise aus FitnessArmbändern oder auch Sensoren zur Blutzuckermessung, gewinnt. Somit besteht 
die Möglichkeit für den Patienten, alle relevanten Daten an einem Ort zu sichern und je nach Bedarf dem medizinischen Fachpersonal zur Verfügung zu stellen.

Durch die Implementierung der medizinischen Notfalldaten, welche der Patient auf freiwilliger Basis auf seiner Gesundheitskarte anlegen kann, wird besonders die Erstversorgung von Notfallpatienten entscheidend verbessert. Durch Auslesung dieser Daten, können Notärzte beispielsweise über chronische Erkrankungen aufgeklärt werden, um auch im Falle eines bewusstlosen Patienten die richtigen Schritte ergreifen zu können. Medikamentenunverträglichkeiten und Allergien können von Anästhesisten zuverlässig eingesehen werden und verringern somit das Risiko von Komplikationen bei einer Notoperation.

Der Deutsche Bundestag überwacht weiterhin die Entwicklung im Bereich des eHealth und überprüft so beispielsweise die Integration von Smartphones und Applikationen im Bereich der Gesundheitskarte, um Patienten ein einfaches und schnelles Management ihrer Gesundheitsdaten zu ermöglichen.

\section{Staatliche Förderungen}

Um die Digitalisierung im Gesundheitswesen auch in Zukunft wegweisend mit zu begleiten, hat der Deutsche Bundestag in den vergangenen Jahren spezielle Förderprogramme bewilligt, welche den Aufbau einer digitalen Gesundheitsinfrastruktur fördert. So stellt das Bundesministerium für Bildung und Forschung (BMBF) beispielsweise im Rahmen des Förderkonzeptes für Medizininformatik Bundesmittel zur Verfügung, um den Datenaustausch zwischen der medizinischen Versorgung, also den Kliniken und Arztpraxen, und der Forschung zu fördern und so eine bessere Reaktion auf Bedürfnisse der Patienten und Versorgungsleistern zu ermöglichen.

Auch durch die Förderung von Big-Data-Zentren leistet das BMBF einen Beitrag, um die Analyse großer Mengen an Gesundheitsdaten schnell und effizient durchführen zu können und somit beispielsweise bisher unbekannte Interdependenzen zu erkennen.

Weiterhin fließen Bundesmittel in die Technologieförderung, um die schnelle Implementierung neuer Informations- und Kommunikationstechnologien in das deutsche Gesundheitssystem zu gewährleisten.

\section{Politische Perspektiven}

\section{Medizinische Versorgung im ländlichen Raum}

Während in urbanen Ballungsgebieten die Attraktivität für die Ansiedelung von medizinischem Personal, wie Allgemeinmedizinern und Fachärzten, als hoch beschrieben werden kann, erlebt der ländliche Raum in den letzten Jahrzehnten einen klaren Rückgang der Ärzteversorgung. Auch wenn kommunale und regionale Verwaltungseinheiten versuchen, diesem Trend beispielsweise durch Stipendien und Bezuschussungen entgegenzuwirken, sind dennoch regionale Fachkliniken und Arztpraxen immer häufiger von Schließungen betroffen.

Die Digitalisierung stellt perspektivisch die Lösung dieser medizinischen Unterversorgung dar. So bieten digitale Übertragungen von Echtzeit-Gesundheitsdaten und Video-Sprechstunden die Möglichkeit, als Arzt eine verhältnismäßig große Fläche zu 
versorgen. Regionale Krankenhäuser können durch digitale Observation ihrer Patienten und gemeinsam finanzierte Facharztstellen die fachärztliche Versorgung vor Ort gewährleisten und ersparen ihren Patienten damit lange Anfahrtszeiten.

Auch die Konsultation von Fachärzten per Datenübertragung aus der hausärztlichen Praxis bietet den Patienten einen guten Service bei kurzen Wegen.

Grundvoraussetzung für die digitale Ärzteversorgung im ländlichen Raum ist und bleibt allerdings der Breitbandausbau auf dem Land. Nur durch die Implementierung von hohen Übertragungsraten ist ein konsequenter Ausbau der Telemedizin auf dem Land durchführbar.

Es ist an der Politik, die Hürden für die digitale Patientenversorgung zu senken und gerechte Qualitätsstandards zu definieren, um Ärzten und Patienten eine gute Gesundheitsversorgung zu ermöglichen. Gleichzeitig muss der Breitbandausbau im ländlichen Raum weiter vorangetrieben werden und in unwirtschaftlichen Regionen im Zweifel auch durch öffentliche Mittel bezuschusst werden, damit der ländlichen Bevölkerung keine strukturellen Nachteile zugemutet werden müssen.

Im Bereich der Arzneimittelversorgung wird die Versorgung zukünftig sowohl von Apotheken vor Ort, wie auch von Versandapotheken zu gewährleisten sein. Insbesondere in bevölkerungsarmen Regionen, in denen die Apothekenversorgung nur schwer umsetzbar ist, sind Versandapotheken ein integraler Bestandteil der Medikamentenversorgung.

\section{Rechtliche Standards für mobile Applikationen}

In der heutigen Zeit, nutzen immer mehr Bürgerinnen und Bürger ein Smartphone im täglichen Gebrauch. Von E-Mails, über soziale Netzwerke, bis zu GesundheitsApps werden diverse Dienste heute über das Handy abgewickelt. Es ist daher wichtig, Standards zu entwickeln, welche die durch diese Dienste gesammelten Daten rechtlich einwandfrei zuordnen.

Insbesondere die Gesundheitsdaten der Nutzer sind in diesem Rahmen gesondert zu betrachten.

Die Anzahl der Applikationen, welche Daten aus Fitness Armbändern, Sensoren und ähnlichem sammeln, nimmt rasant zu. Um einen einheitlichen Qualitätsstandard zu gewährleisten, der diese Daten vergleichbar macht und so auch eine medizinische Nutzbarkeit garantiert, sollte die Politik darauf hinwirken, dass die Daten, welche diese Applikationen sammeln auf einheitlichen Vorgaben beruhen. Besonders im Rahmen des Krankheitsmanagements, wie beispielsweise der Blutzuckerkontrolle, müssen sich Patienten und Ärzte auf die einwandfreie Funktionalität der Applikationen verlassen können.

Weiterhin sind die Datenschutzrichtlinien für Applikationen, welche mit sensitiven Daten, wie Gesundheitsdaten operieren, genauestens zu überwachen. Wie auch im Rahmen des eHealth-Gesetzes für die Gesundheitskarte bereits implementiert, muss hier den Nutzern der Applikationen die vollständige Datensicherheit garantiert werden. Nutzer haben selber zu entscheiden, welche Daten, sie wie verwerten wollen und dürfen nicht durch irgendwelche Hintertüren um ihre Daten betrogen werden. Die Politik hat in diesem Fall eindeutige Maßnahmen zu entwickeln, welche Datendiebstahl sanktionieren. 


\section{Fazit}

Die Digitalisierung im Gesundheitswesen bietet für die Patienten eine Vielzahl von Möglichkeiten, um die eigene Gesundheitsversorgung bewusst zu gestalten und gemeinsam mit Ärzten zukunftsfähige Behandlungsformen zu entwickeln. Die elektronische Gesundheitskarte, welche im Rahmen des eHealth-Gesetzes eingeführt wurde, bietet die richtige Plattform zur Interaktion zwischen Ärzten und Patienten bei gleichzeitiger Datenhoheit des Patienten. Durch direkt abrufbare Informationen verkürzen sich Wartezeiten, Interdependenzen können effizienter erkannt werden und die Notfallversorgung wird entscheidend erleichtert. Der Bund fördert diverse Programme, um die Digitalisierung im Gesundheitswesen zukunftsfähig zu gestalten und Innovationen zu fördern.

Die Folgen der Digitalisierung im Gesundheitswesen sind vielfältig und werden das Gesundheitssystem nach Berechnungen der PWC-Tochter Strategy \& in Zukunft um circa 39 Milliarden Euro jährlich entlasten. Dies wäre ein Rückgang in Höhe von zwölf Prozent des Gesundheitsetats.

Auch für den ländlichen Raum bietet die Digitalisierung vielfältige Möglichkeiten um ländliches Leben und gute Ärzteversorgung wieder vereinbar zu machen.

Die Politik hat hier auch in den kommenden Jahrzehnten die richtigen Weichen zu stellen und Investitionen zu tätigen. Wichtig ist und bleibt, dass gemeinsam und im Austausch mit den Vertretern der Patienten, der Ärzte, der Krankenkassen, aber auch der Industrie und der Apotheken, der Blick in die Zukunft und auf künftige Innovationen gerichtet wird.

Nur durch rechtzeitiges Erkennen von neuen Entwicklungen ist es möglich, frühzeitig die richtigen gesetzlichen Grundlagen zu schaffen, um Innovationen im Gesundheitswesen zu ermöglichen.

Wenn dieser Austausch auch in Zukunft gelingt, wird das Gesundheitswesen in der Bundesrepublik Deutschland auch weiterhin den notwendigen Aufschwung erleben. 